\title{
Square-wave oscillations with different duty cycles
}

\author{
L. Weicker ${ }^{1}$, T. Erneux ${ }^{1}$, O. D'Huys ${ }^{2}$, J. Danckaert ${ }^{2}$, \\ Y. Chembo ${ }^{3}$, M. Jacquot ${ }^{3}$, and L. Larger ${ }^{3}$
}

1.Université Libre de Bruxelles, Optique Nonlinéaire Théorique, Campus Plaine, C.P. 231, 1050 Bruxelles, Belgium 2.Applied Physics Reearch Group (APHY), Vrije Universiteit Brussel, Pleinlaan 2, 1050 Brussels, Belgium 3.UMR CNRS FEMTO-ST 6174, Optics Dept., Univ. of Franche-Comté, 16 Route de Gray, 25030 Besançon, France

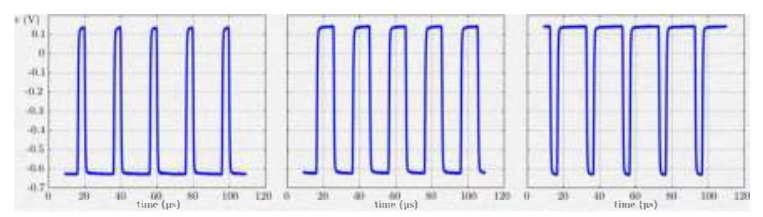

Figure 1: Square-wave oscillations. By changing the feedback phase $\Phi$, the plateau lengths can be tuned.

A fundamental property of nonlinear dynamical systems controlled by a delayed feedback is their tendency to exhibit $2 \tau$-periodic square-wave oscillations of equal plateau lengths ( $\tau$ is the delay) [1]. The question was recently raised whether an optical system could exhibit stable square-wave oscillations with different plateau lengths [2]. We have experimentally found these regimes using a single optoelectronic oscillator (OEO) with a bandpass feedback [3]. See Fig. 1. The period is close to $\tau$ (and not $2 \tau$ ). The response of the OEO is accurately described by the following delay differential equations (time $s=t / \tau$ ) [4]

$$
y^{\prime}=x, \varepsilon x^{\prime}=-x-\delta y+\beta\left[\cos ^{2}(x(s-1)+\Phi)-\cos ^{2}(\Phi)\right] .
$$

Here $\varepsilon=10^{-3}$ and $\delta=8.43 \times 10^{-3}$ are fixed parameters. Stable long time 1-periodic square-wave solutions have

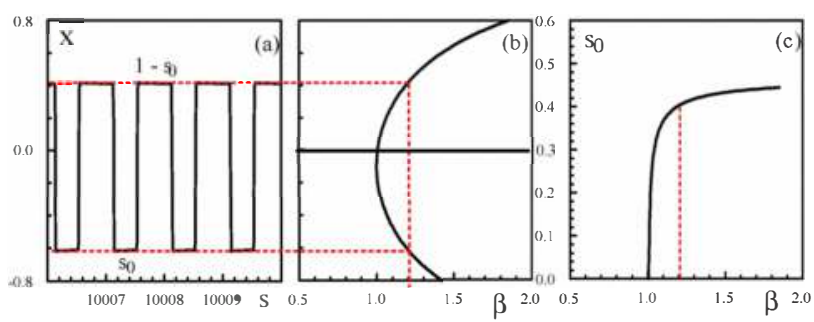

Figure 2: Numerical solution for $\Phi=-\pi / 4+0.1, \beta=1.2$ and analytical bifurcation diagrams.

been obtained numerically (see Fig. 2a). Taking advantage of the small values of $\varepsilon$ and $\delta$, we have determined analytically the bifurcation diagram of these square-waves. Fig. $2 \mathrm{~b}$ and Fig. 2c show the extrema of $x$ and the length of the shortest plateau as a function of $\beta$. As $s_{0} \rightarrow 0$, the solution disappears through a bifurcation point. We have found numerically that this point is not connected to the Hopf bifurcation points of the zero solution but is an isolated bifurcation point.

1. T. Erneux, "Applied Delay Differential Equations", Springer, Berlin (2009)

2. D.W. Sukow, A. Gavrielides, T. Erneux, B. Mooneyham, K. Lee, J. McKay, and J. Davis, Phys. Rev. E 81, 025206(R) (2010)

3. Y. Chembo Kouomou P. Colet, L. Larger and N. Gastaud, Phys. Rev. Lett. 95, 203903 (2005)

4. M. Peil, M. Jacquot, Y. Kouomou Chembo, L. Larger, and T. Erneux, Phys. Rev. E 79, 026208 (2009) 